\title{
Ultraviolet pulsed laser irradiation of multi-walled carbon nanotubes in nitrogen atmosphere
}

\author{
Ángel Pérez del Pino, ${ }^{1, a)}$ Enikö György, ${ }^{1,2}$ Laura Cabana, ${ }^{1}$ Belén Ballesteros, ${ }^{3}$ \\ and Gerard Tobias ${ }^{1}$ \\ ${ }^{1}$ Instituto de Ciencia de Materiales de Barcelona, Consejo Superior de Investigaciones Científicas \\ (ICMAB-CSIC), Campus UAB, 08193 Bellaterra, Spain \\ ${ }^{2}$ National Institute for Lasers, Plasma and Radiation Physics, P. O. Box MG 36, 76900 Bucharest V, Romania \\ ${ }^{3}$ ICN2_-Institut Catala de Nanociencia i Nanotecnologia, Campus UAB, 08193 Bellaterra, Barcelona, Spain
}

(Received 7 November 2013; accepted 28 January 2014; published online 3 March 2014)

\begin{abstract}
Laser irradiation of randomly oriented multi-walled carbon nanotube (MWCNT) networks has been carried out using a pulsed Nd:YAG UV laser in nitrogen gas environment. The evolution of the MWCNT morphology and structure as a function of laser fluence and number of accumulated laser pulses has been studied using electron microscopies and Raman spectroscopy. The observed changes are discussed and correlated with thermal simulations. The obtained results indicate that laser irradiation induces very fast, high temperature thermal cycles in MWCNTs which produce the formation of different nanocarbon forms, such as nanodiamonds. Premelting processes have been observed in localized sites by irradiation at low number of laser pulses and low fluence values. The accumulation of laser pulses and the increase in the fluence cause the full melting and amorphization of MWCNTs. The observed structural changes differ from that of conventional high temperature annealing treatments of MWCNTs. (C) 2014 AIP Publishing LLC. [http://dx.doi.org/10.1063/1.4864776]
\end{abstract}

\section{INTRODUCTION}

The advances in synthesis techniques have allowed the integration of carbon nanotubes (CNT) in many industrial applications as energy, electronics, biomedicine, or environmental areas. ${ }^{1}$ In such one-dimensional systems, defects play a major role and can even dominate the physical and chemical properties of CNTs. For instance, the response of chemical sensors composed of CNT networks is mainly determined by the chemical reactivity of their defects and tube-tube junctions. ${ }^{2,3}$ Structural and chemical modification and defect creation in CNTs for the enhancement of their functional properties can be accomplished after their synthesis. A main route towards the structural transformation of CNTs goes via high thermal annealing processes. ${ }^{4}$ However, these processes are energy and time consuming, and they are not compatible with the use of polymeric substrates which decompose at relatively low temperature.

On the other hand, laser processing of materials can be compatible with the use of polymeric substrates since it is a surface process. It is known that laser radiation can induce a wealth of physical and chemical mechanisms, usually far from the thermodynamic equilibrium, in materials provoking phase transitions not achievable with conventional methods. ${ }^{5}$ Due to the rapid and versatile nature of laser techniques, they have been used for the development of micropatterns in CNT films, ${ }^{6}$ deposition of CNT-based electrode coatings, ${ }^{7}$ and laser purification and cutting of CNTs by irradiation in oxidative environments. ${ }^{8-10}$ Most reports about laser modification of CNTs employ IR and visible radiation. However,

\footnotetext{
a) Author to whom correspondence should be addressed. Electronic mail: aperez@icmab.es. Tel.+34935801853.
}

UV radiation, which is more likely absorbed by CNTs, may more efficiently provoke structural transformations to the nanotubes. To our best knowledge, just a few reports have focused on UV irradiation of CNTs, which were performed in $\mathrm{Ar}$ atmosphere and in air. ${ }^{11,12}$ Experiments in nitrogen environment have not been reported yet. Nitrogen is an inert gas which might act as nitriding agent under certain conditions. Recently, UV radiation has been also used for the processing and immobilization of CNTs onto solid substrates by means of Matrix Assisted Pulsed Laser Evaporation (MAPLE) method in vacuum. ${ }^{13,14}$

In this work, we study the structural modification of multi-walled carbon nanotubes (MWCNT) induced by ns$\mathrm{UV}$ laser irradiation in an inert $\mathrm{N}_{2}$ gas atmosphere. We demonstrate that the observed processes are different from those taking place during conventional thermal annealing. Thermal simulations show that laser pulses induce very short and intense thermal cycles in the CNTs during the laser irradiation that could account for the observed changes in the structure of the CNTs.

\section{EXPERIMENTAL}

\section{A. Deposition of MWCNT films}

Multi-walled carbon nanotube samples were supplied by Thomas Swan \& Co. Ltd as a dry powder. The impurities present in the samples include amorphous carbon, graphitic particles (carbonaceous crystalline materials with few graphitic layers), and some metal particles. The metal particles, from the catalyst used for the MWCNT synthesis, are covered by graphitic shells which inhibit their dissolution during a simple acid wash. Therefore, the as-received MWCNT material was steam purified before a $\mathrm{HCl}$ wash, following a 
previously reported protocol. ${ }^{15}$ Briefly, MWCNTs were ground with an agate mortar and pestle, and placed into a silica tube (about $40 \mathrm{~mm}$ diameter) which was then introduced into the alumina tube of the furnace. Steam was introduced by bubbling argon $\left(150 \mathrm{ml} \mathrm{min}^{-1}\right)$ through a flask containing hot water $\left(98^{\circ} \mathrm{C}\right)$. The whole system was initially purged with argon for $2 \mathrm{~h}$ to ensure the complete removal of oxygen, before heating the furnace to $900^{\circ} \mathrm{C}$ during $1.5 \mathrm{~h}$ to allow the purification of the material. This treatment is known to remove the amorphous carbon present in the sample and the graphitic shells that are covering the metal particles. The solid powder was collected and treated with $\mathrm{HCl}$ to remove the now exposed metal nanoparticles. ${ }^{16}$ The purified MWCNTs were collected by filtration through a $0.2 \mu \mathrm{m}$ polycarbonate membrane and thoroughly washed with water until neutral $\mathrm{pH}$.

A dispersion of MWCNTs (0.1 wt. \%) in chloroform was prepared ${ }^{17}$ by bath sonication for $40 \mathrm{~min}$. To remove any agglomerates that could be present in the dispersion, the solution was filtered through glass fibre. Afterwards, MWCNT layers were prepared by drop drying, depositing $200 \mu \mathrm{l}$ of dispersion onto a silicon substrate. During the deposition process, the substrate was heated to aid the evaporation of the solvent.

\section{B. Laser irradiation of MWCNT films}

Laser processing of MWCNT layers was carried out using a Q-switched Nd:YAG laser system (model Brilliant from Quantel). The experiments were performed at $266 \mathrm{~nm}$ wavelength by joining $2 \omega$ and $4 \omega$ harmonic oscillators to the primary system. The pulse duration was about $3 \mathrm{~ns}$ (FWHM), with Gaussian-like temporal evolution, and the pulse repetition rate was set to $10 \mathrm{~Hz}$. The laser beam spatial distribution was transformed from nearly Gaussian to homogeneous squared shape by means of a Galilean telescope and a squared mask. Next, the laser beam was focussed by means of a $\mathrm{CaF}_{2}$ lens onto the samples, located inside a vacuum chamber, being the irradiated area $1 \times 1 \mathrm{~mm}^{2}$. Larger $1 \times 1 \mathrm{~cm}^{2}$ surfaces were processed by irradiating adjacent sites with a separation distance of $1 \mathrm{~mm}$. The subsequent processing was carried out in $\mathrm{N}_{2}$ gas environment at $2 \times 10^{4} \mathrm{~Pa}$ pressure. The experiments were performed inside a stainless steel chamber, evacuated to a residual pressure of $10^{-4} \mathrm{~Pa}$. The laser fluence (total energy of the pulse divided by the beam area on the sample surface) was set to values in the range of $40-100 \mathrm{~mJ} \mathrm{~cm}^{-2}$ and the number of subsequent laser pulses between 10 and 500 .

The morphology of the laser processed samples was characterized by field emission scanning electron microscopy (FESEM) by means of a QUANTA-FEI 200 system. The study of the structure of the irradiated MWCNTs at the nanoscale was investigated by high resolution transmission electron microscopy (HRTEM) with a FEI Tecnai G2 F20 HRTEM operated at $200 \mathrm{kV}$. The TEM specimens were prepared by gently scratching the sample's surface to release the carbon nanotubes. Afterwards, the material was dispersed in $\mathrm{EtOH}$, sonicated, and placed dropwise onto a holey carbon copper support grid for HRTEM observation. Raman spectroscopy analyses were performed by means of a LabRAM 800 system from Horiba Jobin Yvon. The laser wavelength and power for the measurements were, respectively, chosen to $532 \mathrm{~nm}$ and $0.7 \mathrm{~mW}$. The laser spot size onto the samples surface was about $1-2 \mu \mathrm{m}^{2}$. Chemical mapping of the samples was performed with a confocal Raman microscope alpha300R from WITec, using a $532 \mathrm{~nm}$ laser wavelength. $75 \times 75$ spectra were acquired in squared $10 \times 10 \mu \mathrm{m}^{2}$ surface regions, integrating $100 \mathrm{~ms}$ per spectrum.

\section{RESULTS}

\section{A. Morphology of the films}

The morphology of the films prior and after laser irradiation was investigated by SEM (Figure 1). The deposited MWCNT films show a porous and rough morphology, with a thickness that varies from few up to tens of $\mu \mathrm{m}$ across the
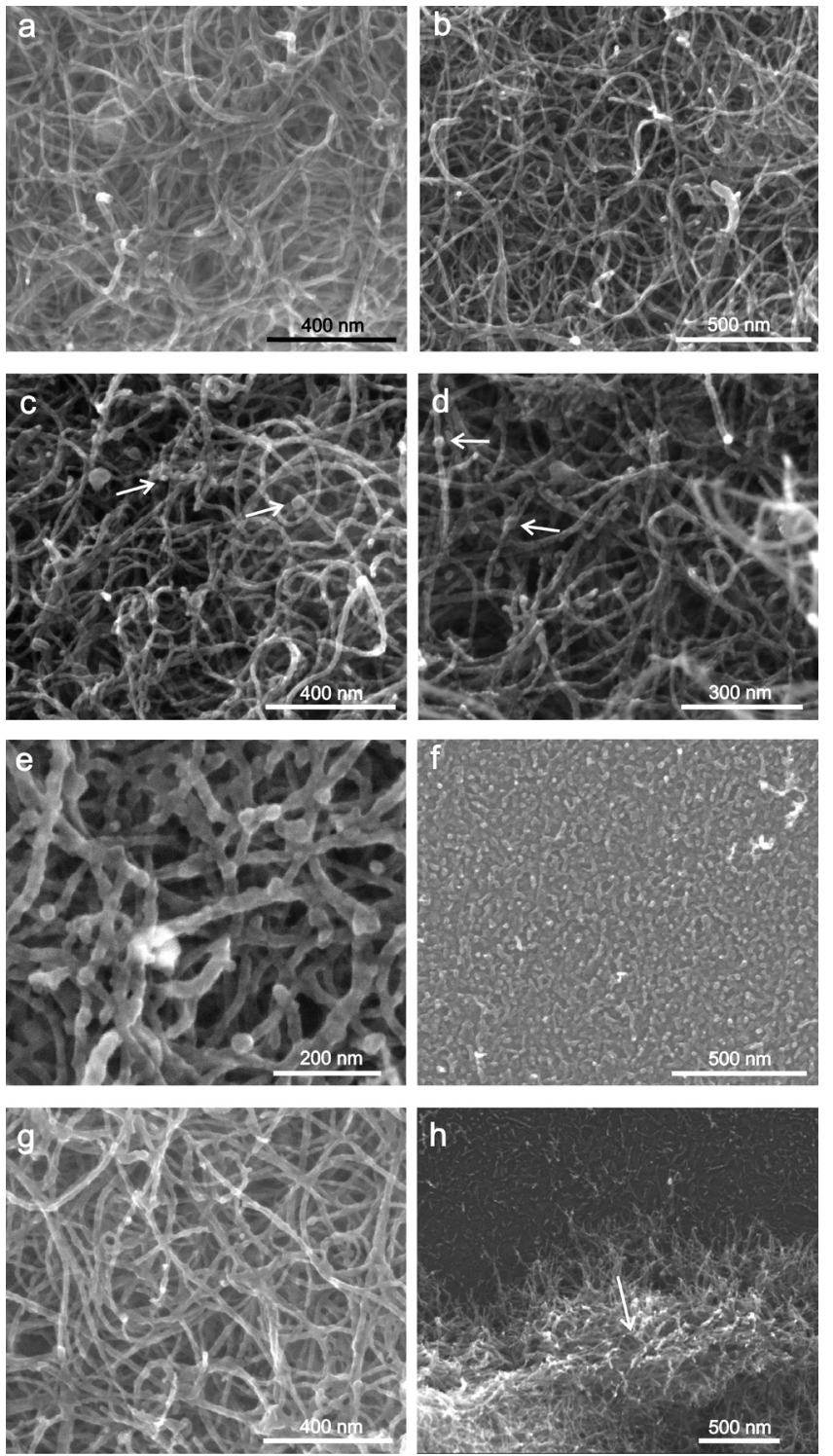

FIG. 1. SEM images of (a) non-irradiated MWCNTs, and MWCNTs irradiated with $40 \mathrm{~mJ} \mathrm{~cm}^{-2}$ and (b) 10 , (c) 100 , and (d) 500 pulses; samples irradiated with (e) $60 \mathrm{~mJ} \mathrm{~cm}^{-2}$ and 500 pulses, (f) $80 \mathrm{~mJ} \mathrm{~cm}^{-2}$ and 500 pulses, and $100 \mathrm{~mJ} \mathrm{~cm}^{-2}$ with an accumulation of (g) 10 and (h) 100 pulses. Arrows in (c) and (d) indicate nano-sized droplets. Arrow in (h) points to a partially molten MWCNT "island" surrounded by molten-like film. 
surface. The films are composed of a randomly oriented network of carbon nanotubes, about $10-30 \mathrm{~nm}$ in diameter and a large fraction are about $1 \mu \mathrm{m}$ in length (Figure 1(a)). The asdeposited nanotubes present a smooth outer surface and exhibit both straight and bent shapes. The observed void space between CNTs can be considerable, up to hundreds of nm, and varies along the film. The overlapping degree between CNTs also changes alongside the nanotube tangled-film, though it is small (up to tens of nm). A scarce number of bundles composed of parallel nanotubes can be observed. The CNTs irradiated with the lowest fluence and shortest time (10 pulses and $40 \mathrm{~mJ} \mathrm{~cm} \mathrm{~cm}^{-2}$ ) show the same aspect as the non-irradiated ones (Figure 1(b)). However, when the number of pulses is increased to 100 and 500, the formation of protuberances, about few nm in size, becomes evident at the CNT surface (Figures 1(c) and 1(d)). The number and size of the protuberances increases with the number of accumulated pulses. In addition, in some areas, the CNTs start to present a molten aspect and the formation of tens of nm quasi-spherical droplets (shown by arrows in Figures 1(c) and 1(d)). Laser irradiation with a higher laser fluence of $60 \mathrm{~mJ} \mathrm{~cm}^{-2}$ produces a similar effect in the material but the process becomes more noticeable when compared with the samples obtained with $40 \mathrm{~mJ} \mathrm{~cm}^{-2}$ with the same number of pulses. Moreover, the samples irradiated with 500 pulses show areas in which the nanotubes present a completely molten aspect (Figure 1(e)). The contour of the nanotubes also shows droplet-like structures about tens of $\mathrm{nm}$ in size. Though the CNTs still present their long shape, their diameter slightly increases, up to about $50 \mathrm{~nm}$ in some places, and the CNTs appear welded between them. This effect is even greater in the samples irradiated with $80 \mathrm{~mJ} \mathrm{~cm}^{-2}$ and, after 500 accumulated pulses, hundreds of $\mu \mathrm{m}$ sized areas appear as a continuous and rough meander-like film probably composed of molten and re-solidified material (Figure 1(f)). Finally, the MWCNTs

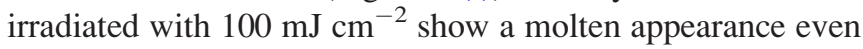
after just 10 pulses (Figure 1(g)). An increase in the accumulated number of pulses leads to the formation of a large-size continuous molten-like film, though some micrometric "islands" composed of partially molten nanotubes are also visible (shown by an arrow in Figure 1(h)). We note that no signs of extensive vaporization of the MWCNTs were identified in any of the irradiated samples since neither craters nor CNTs' fragments were observed in the irradiated films. Therefore, as a general rule, the effect of the laser radiation in the MWCNTs augments with the increase in the laser fluence and the accumulated number of pulses.

\section{B. Structural analyses}

Raman spectroscopy measurements of the as-prepared as well as the films irradiated with $100 \mathrm{~mJ} \mathrm{~cm}^{-2}$ laser fluence are depicted in Figure 2(a). The spectra of the "island" regions consisting of partially molten MWCNTs show dominating bands centered at around $1350 \mathrm{~cm}^{-1}$ (D) and $1590 \mathrm{~cm}^{-1}(\mathrm{G})$, in addition to second-order bands centered at about $2690\left(\mathrm{G}^{\prime}\right)$ and $2940 \mathrm{~cm}^{-1}(\mathrm{D}+\mathrm{G}) .{ }^{18}$ The contribution of less intense and broader bands is also observed at about $1100 \mathrm{~cm}^{-1}, 2450 \mathrm{~cm}^{-1}$, and $3240 \mathrm{~cm}^{-1}$ (2 G). Conversely, regions coated with continuous molten-like material show very weak CNT bands, being practically D and G the only visible spectral features. Two additional more intense bands associated with the silicon substrate appear at about 520 and $960 \mathrm{~cm}^{-1}$.

The main interest of the Raman analysis is the evolution of the D and G bands of MWCNTs since D band is related to structural defects/disorder. Moreover, the D-to-G intensity ratio $(\mathrm{D} / \mathrm{G})$ is usually used as a figure of merit of the defect content of the CNTs. ${ }^{4}$ A careful inspection of the spectral range corresponding to the $\mathrm{D}$ and $\mathrm{G}$ bands reveals additional features, such as asymmetries and shoulders in the $G$ band. According to Maldonado et al., ${ }^{19}$ this region was fit using five Gaussian bands: I $\left(\sim 1190 \mathrm{~cm}^{-1}\right), \mathrm{D}\left(\sim 1350 \mathrm{~cm}^{-1}\right), \mathrm{D}^{\prime \prime}$ $\left(\sim 1480 \mathrm{~cm}^{-1}\right), \quad \mathrm{G} \quad\left(\sim 1590 \mathrm{~cm}^{-1}\right)$, and $\mathrm{D}^{\prime}\left(\sim 1620 \mathrm{~cm}^{-1}\right)$ (Figure 2(b)). The presence of I band was previously associated with the appearance of disordered graphitic carbons and with the incorporation of nitrogen to CNT structure. ${ }^{20}$ The existence of $\mathrm{D}^{\prime}$ and $\mathrm{D}^{\prime \prime}$ features could be induced by irregular $\mathrm{d}_{002}$ spacing and stacking defects of the concentric graphene layers which form MWCNTs. ${ }^{19}$ As it can be observed in Figures 2(a) and 2(b), the intensity of the D band is higher than that of the $\mathrm{G}$ band in the non-irradiated CNTs. However, as a general behaviour, the accumulation of laser pulses provokes the decrease in the D band intensity which tends to match the $\mathrm{G}$ band. In particular, the reduction of the $\mathrm{D} / \mathrm{G}$ ratio is remarkable when accumulating laser pulses with $100 \mathrm{~mJ} \mathrm{~cm}^{-2}$. Below $80 \mathrm{~mJ} \mathrm{~cm}^{-2}$, the $\mathrm{D} / \mathrm{G}$ ratio is practically constant with fluence after the application of just 10 laser pulses, but it decreases upon irradiation at this laser fluence. Taking into account that at $80 \mathrm{~mJ} \mathrm{~cm}^{-2}$ laser fluence, melting of the CNTs begins to be substantial as revealed by SEM inspections, the decrease in $\mathrm{D} / \mathrm{G}$ ratio seems to be related to the laser-induced melting of the CNTs. The obtained $D^{\prime} / G$ intensity quotient of the studied MWCNTs shows a behaviour with the laser fluence and number of accumulated pulses qualitatively analogous to that of $D / G$ (Figures 2(c) and 2(d)). However, no clear evolution of $\mathrm{D}^{\prime \prime} / \mathrm{G}$ quotient is observed for $\mathrm{D}^{\prime \prime}$ bands which appear to be less intense and wider than the corresponding $\mathrm{D}^{\prime}$ bands. $\mathrm{I} / \mathrm{G}$ ratio seems to slightly decay with the laser fluence and number of accumulated pulses. However, the large experimental error associated with the weak and wide I band induces a high uncertainty in these measurements.

Raman maps were also acquired in several $\mu \mathrm{m}^{2}$ regions of the obtained samples revealing structural differences in the micrometric spatial range. Figure 3 shows the Raman map obtained in a partially molten MWCNT island surrounded by continuous molten-like film in the sample irradiated with $100 \mathrm{~mJ} \mathrm{~cm}^{-2}$ and 500 laser pulses. As a general feature, the spectra display low intensities which are even weaker in the molten film part than inside the island. Furthermore, the position of the $\mathrm{G}$ band appreciably shifts to higher wavenumbers and a slight increase in the D-to-G band intensity ratio is also observed from the inner part of the island to the outer region, where the CNTs are completely molten and resolidified.

The structure of the MWCNTs was next studied through HRTEM. Non-irradiated nanotubes show concentric shells 

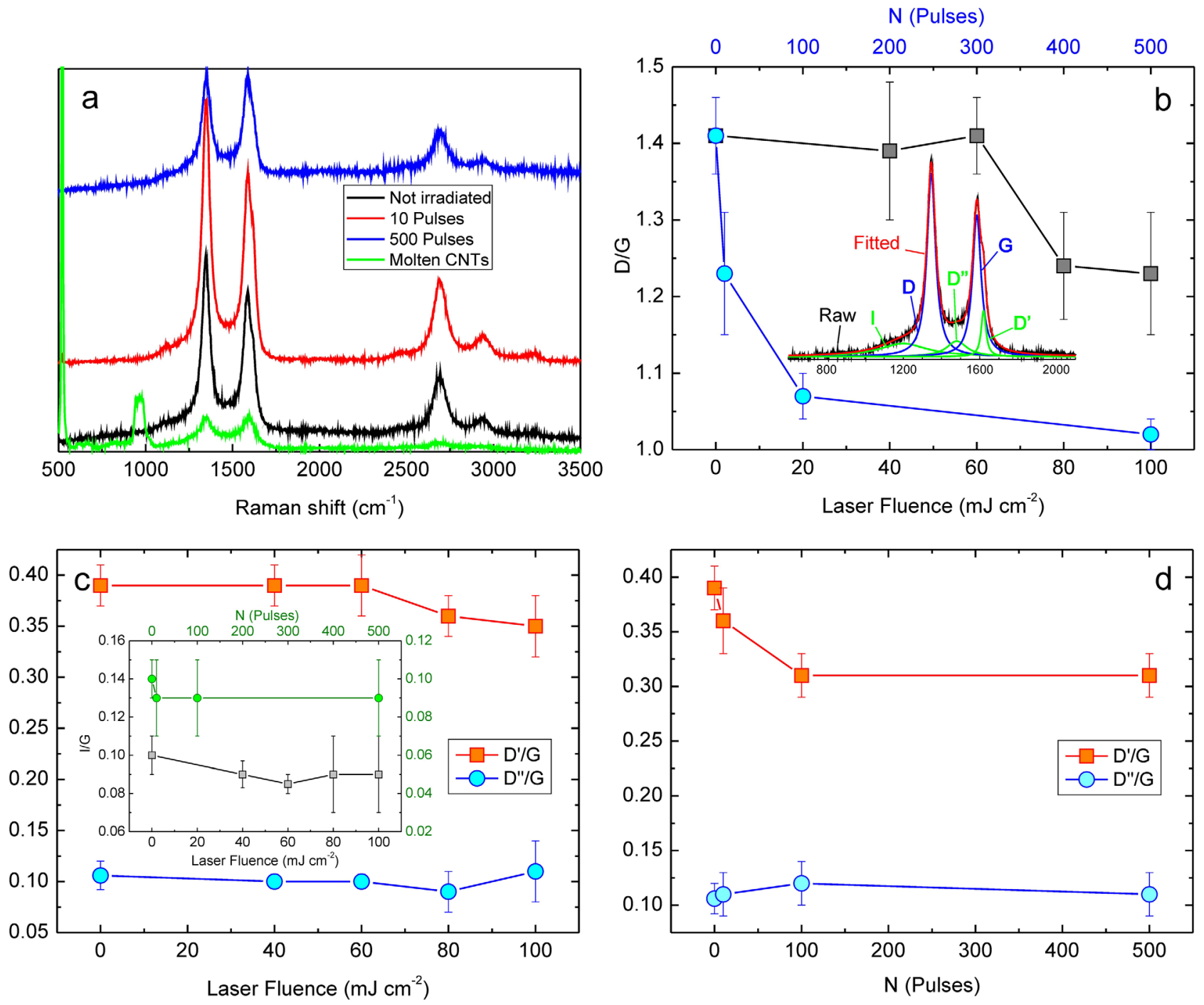

FIG. 2. (a) Typical Raman spectra obtained in samples irradiated with $100 \mathrm{~mJ} \mathrm{~cm}^{-2}$. (b) $\mathrm{D} / \mathrm{G}$ ratio as a function of laser fluence after the accumulation of 10 pulses (ם) and as a function of accumulated number of pulses in sample irradiated with $100 \mathrm{~mJ} \mathrm{~cm}^{-2}(\bullet)$. Inset: example of fitting curves in MWCNT spectrum. (c) $\mathrm{D}^{\prime} / \mathrm{G}$ and $\mathrm{D}^{\prime \prime} / \mathrm{G}$ ratios as a function of laser fluence after 10 pulses. Inset: $\mathrm{I} / \mathrm{G}$ ratio as a function of laser fluence after 10 pulses ( $\left.\mathbf{\square}\right)$ and as a function of accumulated number of pulses in samples irradiated with $100 \mathrm{~mJ} \mathrm{~cm}^{-2}(\bullet)$. (d) $\mathrm{D}^{\prime} / \mathrm{G}$ and $\mathrm{D}^{\prime \prime} / \mathrm{G}$ ratios as a function of accumulated number of pulses in samples irradiated with $100 \mathrm{~mJ} \mathrm{~cm}^{-2}$.

(Figure 4(a)) with the expected $0.34 \mathrm{~nm}$ spacing between them. Most of the CNTs are straight or somewhat curved from tens to hundreds of $\mathrm{nm}$ distance, and some of them appear twisted. Most of the nanotubes exhibit a low degree of defects. However, distorted or incomplete graphitic shells, irregular spacing, and slip planes can be observed in some locations. Most of the nanotubes are open-ended, as a consequence of the steam treatment employed for their purification, and large areas of amorphous material are not observed attached to or into the nanotube structure. Nevertheless, the investigations revealed notable modifications of the MWCNT shell structure as a consequence of the laser irradiation (Figures 4(b), 5, and 6). Many of the observed CNTs show bulges, up to few tens of $\mathrm{nm}$ in size, at specific locations (Figure 4(b)). These protrusions can be recognized as the grains and droplets observed by SEM in the MWCNTs irradiated with low laser fluences and high number of accumulated pulses (Figures 1(c) and 1(d)). High resolution observation of the protuberances mainly shows two different types of formation: bulges with irregular shape and amorphous nature (Figure 4(b)), and more compact grains composed of nmsized zones with well-defined atomic planes (Figure 5). Selective fast Fourier transform (FFT) of the crystalline zones reveals lattice parameters of about $0.18,0.20$, and $0.25 \mathrm{~nm}$ which can be assigned to $\langle 200\rangle,\langle 111\rangle$, and $\langle 110\rangle$ crystallographic orientations of cubic diamond. ${ }^{21}$ Crystals with lattice parameters of around 0.22 and $0.27 \mathrm{~nm}$, which could be attributed to distorted diamond, were also observed. Most of the diamond crystals were observed in the outer regions of the CNTs and the neighbouring material of the crystalline regions typically presents an amorphous aspect. The shells of this type of nanotubes appear full of defects which delimit them to nm-long fragments. The layers' fragments also show a noticeable disordered nature. On the other hand, CNTs with highly corrugated shell structure were also distinguished (Figures 6(a) and 6(b)). This type of nanotubes exhibits shell undulations in the range of 5 to tens of $\mathrm{nm}$ which, in some cases, only appear in one side of the CNTs. In addition, the 


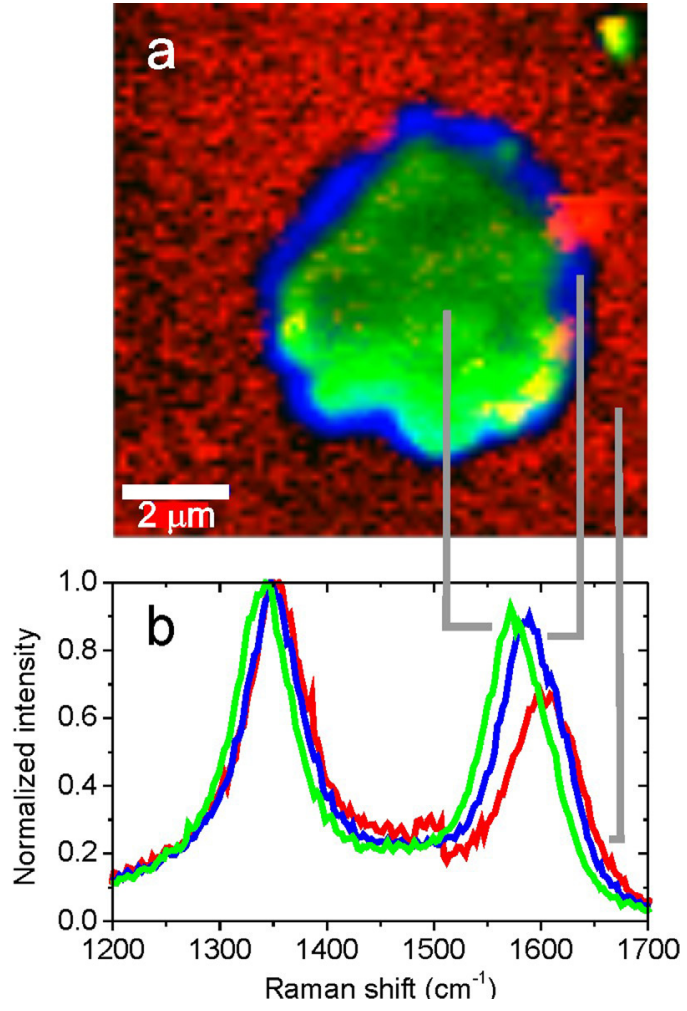

FIG. 3. (a) Colour coded Raman map of the sample irradiated with $100 \mathrm{~mJ} \mathrm{~cm}^{-2}$ and 500 pulses, and (b) typical Raman spectra measured at different positions in which colours correspond to image (a).
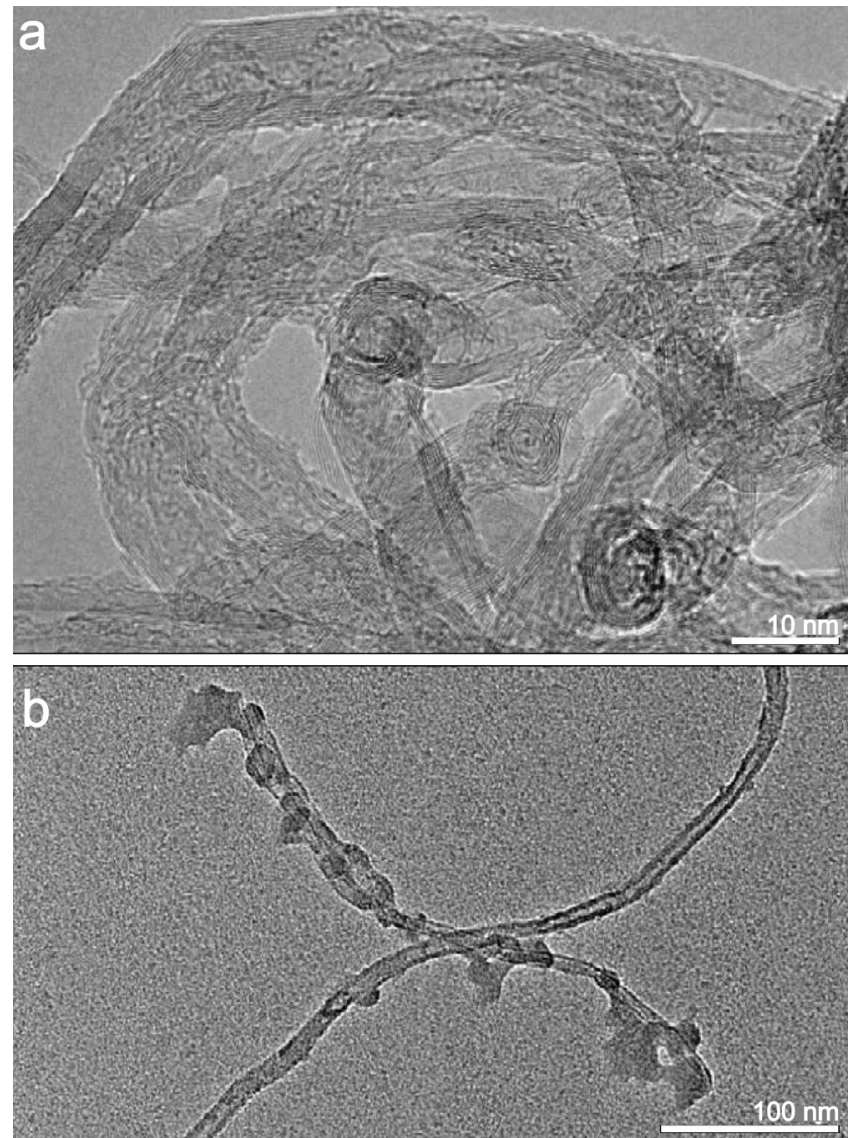

FIG. 4. TEM images of MWCNTs (a) as-deposited and (b) irradiated with laser showing protuberances. shells reveal discontinuities, distortions, and irregular spacing in a number of locations. Despite the high curvature of the shells, their layered structure is preserved along tens of $\mathrm{nm}$. Besides, the inner hollow cavity of the CNTs shows nested walls at several locations which segment the nanotubes in bamboo-like fashion (Figure 6(b)). Amorphization of the ends of the MWCNTs modified by laser radiation is also visible (not shown). Finally, material with amorphous structure was also detected (Figure 6(c)). The material usually appears as tens of nm broad lengthened fragments wider than initial MWCNTs, many times attached to round amorphous particles, forming a sort of mesh between them. Detailed inspection of this material reveals, in addition to completely amorphous zones, the presence of nm-sized regions with few graphitic layers (about $0.33 \mathrm{~nm}$ plane spacing) as well as locations in which disordered short-range features are observed. The FFT of some of these disordered nm-sized areas shows blurred geometrical features pointing to a kind of

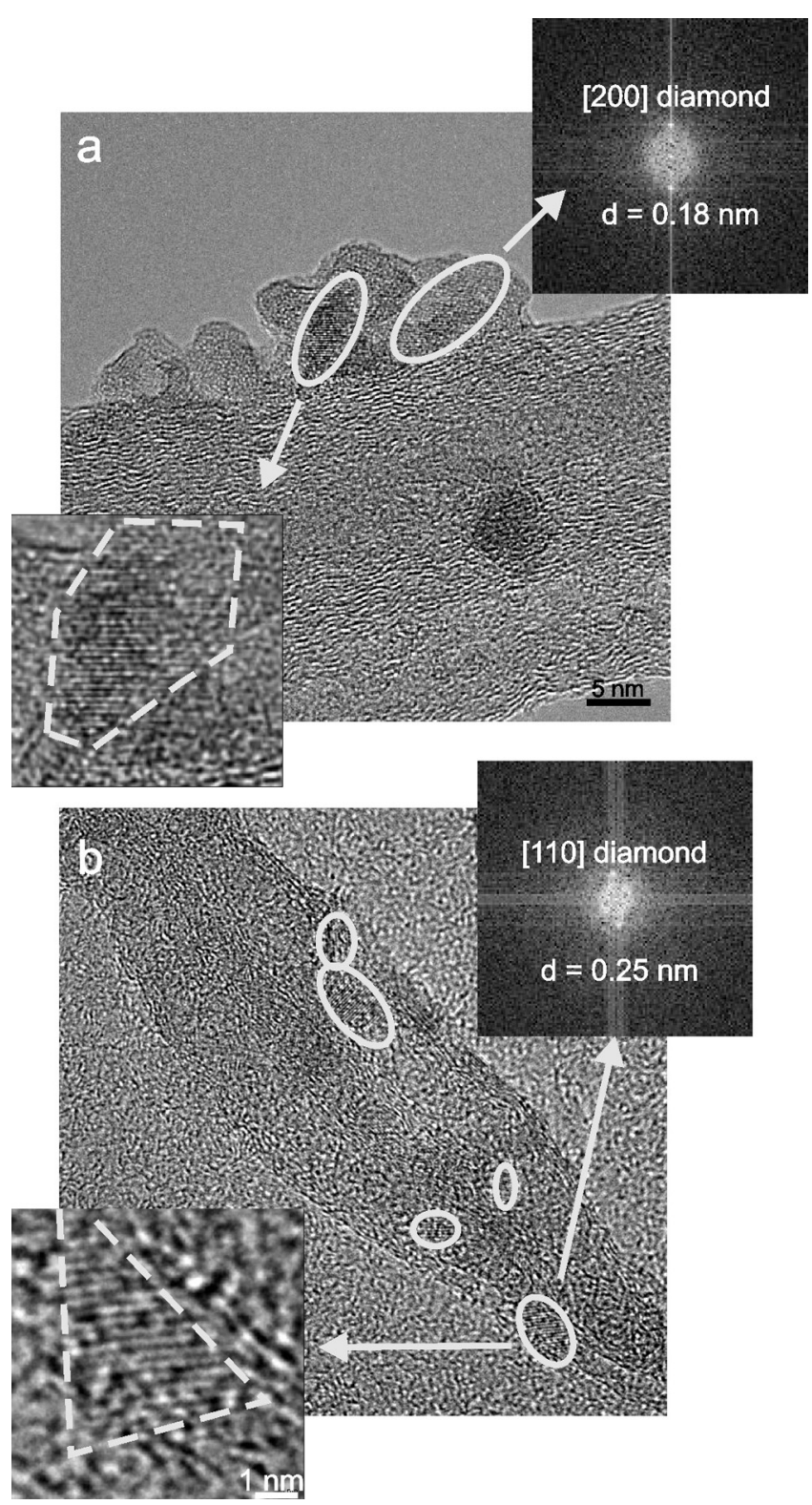

FIG. 5. HRTEM images of irradiated MWCNTs showing the formation of diamond nanocrystals and their corresponding FFTs. 

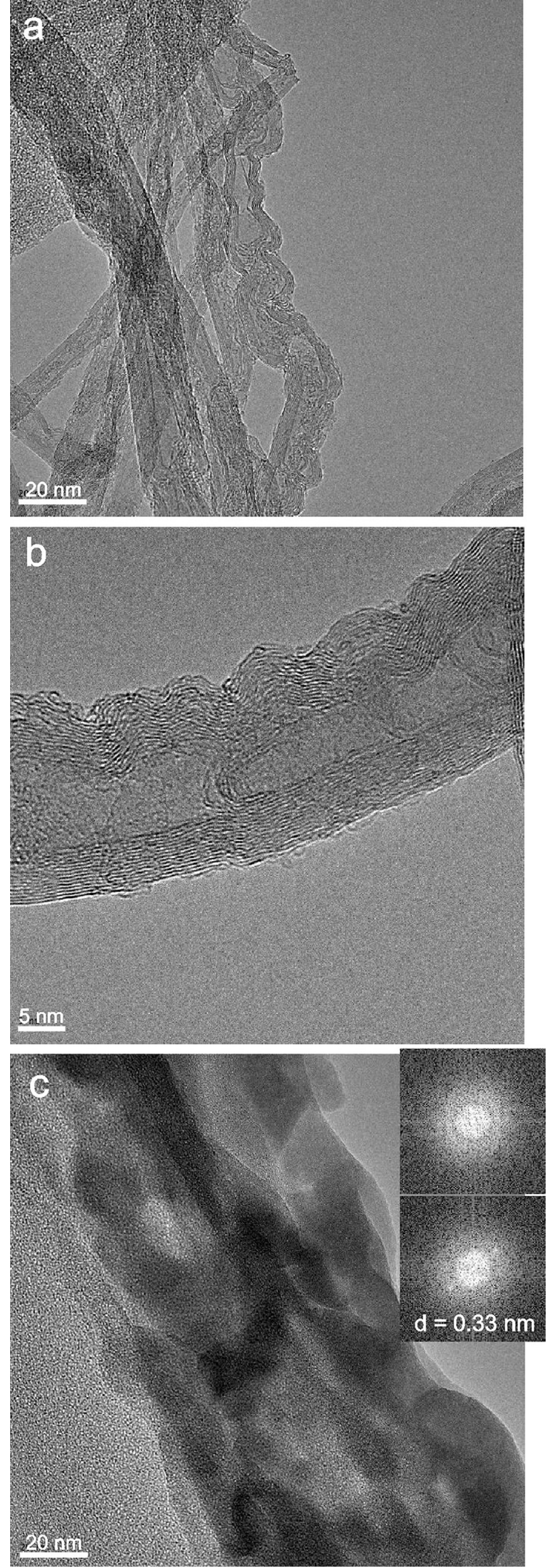

FIG. 6. HRTEM images of (a) and (b) corrugated and (c) amorphous MWCNTs. ordering between atoms (FFT in Figure 6(c)). Due to the morphologic similarities observed by SEM investigations, we can infer that this material is the result of the melting and recrystallization process of the irradiated nanotubes' network (Figures $1(\mathrm{e})$ and $1(\mathrm{~g})$ ). It is worth mentioning that electron energy loss and energy dispersive X-ray spectroscopy analyses did not reveal any detectable amount of nitrogen inserted into the CNT structure.

\section{Thermal simulations}

The temperature evolution of the carbon nanotubes during laser irradiation was simulated considering pure thermal mechanisms since the used photon energy $(4.7 \mathrm{eV})$ is quite lower than the $\mathrm{C}=\mathrm{C}$ dissociation bond energy $(6.4 \mathrm{eV}){ }^{22}$ The calculation was carried out by solving a $2 \mathrm{D}$ transient heat conduction model by means of partial differential equations using COMSOL Multiphysics 4.3 a software. In order to get a general understanding of the irradiation effects in the MWCNT films, various simple geometries were considered for the simulation: (i) continuous films of 1 to $10 \mu \mathrm{m}$ thickness, and (ii) isolated MWCNTs, $1 \mu \mathrm{m}$ length and $15 \mathrm{~nm}$ in diameter. Since the nanotubes are randomly oriented, two idealized configurations, vertical and horizontal, are taken into account in the model. Isolated nanotubes have been studied in both of these configurations: (1) vertical (CNT V) and (2) horizontal (CNT H). In the second case, the horizontal nanotube is sustained by vertical nanotubes at each of its extremes (CNT H) (Figure 7(a)). Up to three horizontal MWCNTs connected vertically (3 CNT V) or horizontally (3 CNT H) were simulated. All these structures were considered to be isotropic and placed on a semi-infinite silicon substrate. The carbon nanotubes were considered solid, without internal shell structure, so its influence in thermal conductivity was not taken into account. Considering a Gaussian-like temporal evolution of the laser pulse, the heat conduction equation solved in all the modelled components is the following:

$$
\rho C_{p} \frac{\partial T}{\partial t}-\nabla\left(k_{t h} \nabla T\right)=\frac{F}{\tau} e^{-4 L n(2)\left(\frac{t \tau \tau}{\tau}\right)^{2}}(1-R) \alpha e^{-\alpha z},
$$

where $\mathrm{T}$ is the temperature, $\mathrm{t}$ is the time, $\mathrm{F}$ is the incident laser fluence, $\tau$ is the laser pulse duration, $\rho$ is the material density, $\mathrm{C}_{\mathrm{p}}$ is the specific heat capacity, $\mathrm{k}_{\mathrm{th}}$ is the thermal conductivity, and $\mathrm{R}$ and $\alpha$ are, respectively, the reflectance and the optical absorption coefficient of the corresponding materials at $\lambda=266 \mathrm{~nm}$. The melting phase transition was also considered in all materials by increasing the specific heat for a temperature range of $1 \mathrm{~K}$ after the melting temperature $\left(\mathrm{T}_{\mathrm{m}}\right)$

$$
C_{p, e}(T)=C_{p}(T)+\frac{L_{m}}{\Delta T} \cdot\left[H\left(T-T_{m}\right)-\mathrm{H}\left(T-T_{m}-1\right)\right],
$$

where $\mathrm{L}_{\mathrm{m}}$ is the specific melting latent heat of the considered material, $\Delta \mathrm{T}=1 \mathrm{~K}$ and $\mathrm{H}(\mathrm{T})$ is the Heaviside function. Thermal leakages due to radiation and convection mechanisms were overlooked. Several considerations need to be 

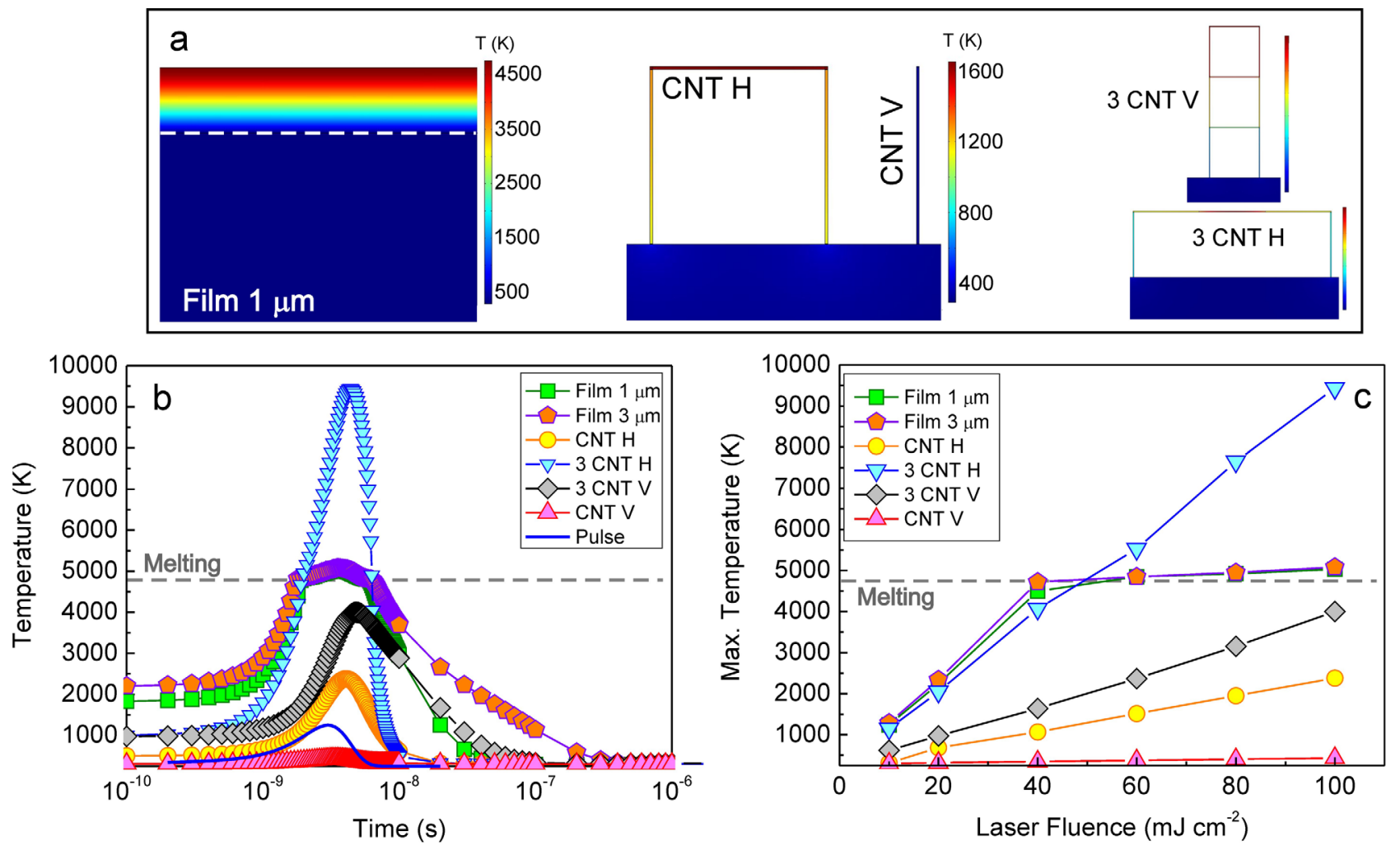

FIG. 7. Simulated temperature distribution in (a) $1 \mu \mathrm{m}$ thick film, and horizontal and vertical nanotubes distributions irradiated with a $100 \mathrm{~mJ} \mathrm{~cm}^{-2}$ laser pulse at $6 \mathrm{~ns}$. The dashed line in the film image displays the film-substrate boundary. (b) Thermal evolution as a function of time in material irradiated with $100 \mathrm{~mJ} \mathrm{~cm}^{-2}$ and (c) maximum temperature reached in the material as a function of laser fluence.

taken into account to perform the calculations. The considered optical and thermophysical parameters of the materials composing the compact film and MWCNTs are detailed in Table I. Many reported works model carbon nanotubes films as continuous solid films. In order to get deeper understanding of the thermal mechanisms involved during the laser irradiation, in this work, the solid film approximation is compared with isolated CNT assessment which could exhibit an important role in highly porous films as those obtained in this work. The continuous solid film of our simulation is considered to be porous so its effective mass density was chosen to a typical value significantly lower than that of MWCNTs themselves. $^{23}$ The mass density of liquid carbon is similar to that of MWCNTs. ${ }^{24}$ The thermal conductivity variation with temperature in the film was considered similar to that of MWCNT yarns, ${ }^{23}$ whereas the value measured at room temperature in Ref. 25 was taken for isolated MWCNTs. The rest of optical and thermophysical properties of the film and isolated CNTs was considered to be the same. The specific heat capacity and thermal conductivity in solid state for
MWCNTs were taken from Ref. 23. Their values after melting were considered to be those of highly ordered pyrolytic graphite (HOPG). ${ }^{26}$ The melting temperature was chosen as the one of perfect single-walled carbon nanotubes (SWCNTs), ${ }^{27}$ though structural defects of real CNTs reduce their melting temperature. The specific melting latent heat value was taken as the one of graphite. ${ }^{28}$ Concerning the optical properties, the reflectance in solid state was valued as that of amorphous carbon films, ${ }^{29}$ whereas after melting was considered similar to the one of HOPG. ${ }^{26}$ The optical absorption at $266 \mathrm{~nm}$ was also estimated as that of HOPG. ${ }^{26}$ Light scattering effects were neglected. It has to be noticed that touching nanotubes are coupled by van der Waals interactions, which leads to an increase in the thermal resistance in tube-to-tube contact regions. Therefore, the thermal transport in MWCNT bundles is expected to be highly influenced by these contact regions. This effect was also taken into account in the model by considering $2 \mathrm{~nm}$ thick tube-to-tube contact regions with reduced thermal conductivity, which was assumed similar to the reported intrinsic value between

TABLE I. Thermal and optical parameters used in numerical calculations.

\begin{tabular}{|c|c|c|c|c|c|c|c|}
\hline & $\rho\left(\mathrm{kg} \mathrm{m}^{-3}\right)$ & $\mathrm{C}_{\mathrm{p}}\left(\mathrm{J} \mathrm{kg} \mathrm{K}^{-1}\right)$ & $\mathrm{k}_{\mathrm{th}}\left(\mathrm{W} \mathrm{mK} \mathrm{mK}^{-1}\right)$ & $\mathrm{T}_{\mathrm{m}}(\mathrm{K})$ & $\mathrm{L}_{\mathrm{m}}\left(\mathrm{J} \mathrm{kg}^{-1}\right)$ & $\mathrm{R}(\%)$ & $\alpha\left(\mathrm{cm}^{-1}\right)$ \\
\hline \multirow[t]{3}{*}{ Film } & 250 (Solid) & & $\mathrm{k}_{\mathrm{th}}(\mathrm{T})($ Solid $)$ & & & & \\
\hline & 1400 (Liquid) & $\mathrm{C}_{\mathrm{p}}(\mathrm{T})$ (Solid) & 90 (Liquid) & & & 10 (Solid) & $81 \times 10^{3}($ Solid $)$ \\
\hline & & 998 (Liquid) & & 4800 & 9800 & 73 (Liquid) & $11 \times 10^{4}$ (Liquid) \\
\hline \multirow[t]{2}{*}{ MWCNT } & 1350 (Solid) & & 3000 (Solid) & & & & \\
\hline & 1400 (Liquid) & & 90 (Liquid) & & & & \\
\hline
\end{tabular}


MWCNT shells $\left(2 \mathrm{~W} \mathrm{mK}{ }^{-1}\right){ }^{30,31}$ The thermophysical parameters of silicon substrate were taken from Ref. 32 .

Thermal simulations reveal that the laser pulses induce thermal cycles in the MWCNTs about tens to a hundred of ns in duration and with heating-cooling rates which can reach up to $10^{12} \mathrm{~K} / \mathrm{s}$ (Figure $7(\mathrm{~b})$ ). The modelled continuous solid films with different thickness show a very similar behaviour. The thermal penetration depth is about $1 \mu \mathrm{m}$ in all the simulated films with thicknesses up to $10 \mu \mathrm{m}$. The temperature evolution is rather similar being the developed temperatures and thermal pulse duration slightly higher as film thickness increases. The melting temperature is reached in the films' surface at about $40-60 \mathrm{~mJ} \mathrm{~cm}^{-2}$ laser fluence (Figure 7(c)). Higher laser fluences lead to a slight increase in the maximum developed temperature, near the melting temperature. The time in which the material remains at, and above, the melting temperature does not exceed $10 \mathrm{~ns}$ in any case. Additional simulations, not shown, were performed considering doubled mass density of the film. These studies revealed very similar results, pointing to a not major influence of this parameter in the temperature fluctuation which may be developed in a real CNT film due to network porosity variations.

The simulated thermal evolution of individual MWCNTs significantly depends on their spatial configuration. Due to their high thermal conductivity, the temperature variation along the CNTs is low and the highest thermal gradients are present in the tube-to-tube contact regions, where the thermal conductivity is considerably lower. As a general behaviour, the maximum temperature of the considered CNT-systems linearly increases with the laser fluence in the studied range. The vertically-aligned CNTs (CNT V) do not significantly contribute to the heating of the CNT structures due to their tiny effective area for absorbing laser radiation. The maximum developed temperature would be around $400 \mathrm{~K}$ when irradiated with $100 \mathrm{~mJ} \mathrm{~cm}{ }^{-2}$ laser fluence pulses. Conversely, the horizontal-aligned MWCNT (CNT $\mathrm{H}$ ), in transversal position respect to the incoming radiation, presents a larger effective absorbing area and it considerably heats up. The generated thermal energy would then flow through the vertical nanotubes to the silicon substrate, which is at room temperature. The maximum temperature reached in this system, about $2400 \mathrm{~K}$ when irradiated with $100 \mathrm{~mJ}$ $\mathrm{cm}^{-2}$ fluence, is achieved in the horizontal-aligned MWCNT. Vertical arrangements of various CNT H systems lead to the development of longer thermal pulses thus reaching higher maximum temperatures on the top CNTs. Particularly, a three cells arrangement ( 3 CNT V) leads to the development of up to $4000 \mathrm{~K}$ in the top horizontal MWCNT, whereas the ones located underneath reach hundreds of $\mathrm{K}$ lower temperatures at each subsequent sublevel. The effect of laser radiation is, however, greater in arrangements of horizontal CNTs. As seen in the structure composed of three horizontal nanotubes ( $3 \mathrm{CNT} \mathrm{H}$ ), the developed temperature in the system reveals a substantial increase with the laser fluence, surpassing the melting point when the structure is irradiated with fluences above $50 \mathrm{~mJ} \mathrm{~cm}{ }^{-2}$. Temperatures as high as $9500 \mathrm{~K}$ could be even reached in this ideal system with laser fluences of $100 \mathrm{~mJ} \mathrm{~cm}^{-2}$. In summary, the thermal simulations point out that higher temperatures will be developed in the material by increasing the horizontal overlapping of nanotubes and film thickness. The MWCNTs can reach thousands of Kelvins in the nanosecond time scale and even their melting point can be achieved irradiating them with high enough laser fluence, in concordance with the SEM and TEM characterization. It is worth noting that similar simulations were performed considering MWCNTs with $30 \mathrm{~nm}$ thickness and the obtained results were practically equal to the ones described above.

\section{DISCUSSION}

The photon energy of the laser radiation used in the experiments is $\sim 4.7 \mathrm{eV}$. This UV radiation excites both $\sigma$ and $\pi$-bonded electrons that constitute the MWCNT structure, probably in addition to $\pi$ plasmons,${ }^{33}$ leading to a substantial heating of the CNTs. Lambert-Beer law applied to $15 \mathrm{~nm}$ thick MWCNTs reveals that about $11 \%$ of the laser radiation is absorbed by them. This fact in combination to the high porosity of the as-prepared nanotube network would lead to a deep radiation penetration into a considerable film thickness, certainly much larger than the corresponding radiation penetration depth in a compact film $(\sim 120 \mathrm{~nm})$. On the other hand, the simulations show that the evacuation of thermal energy in CNTs strongly depends on their spatial orientation and tube-tube overlapping configuration, being radically different to that of continuous films (Figure 7). The large influence of tube-tube overlapping regions in the thermal behaviour of the CNT system further increases the difficulty to model a real network of MWCNTs. Thus, compact and continuous film-like simulations should not be applied in highly porous CNT networks. They could be applicable, with some limitations, to dense films of aligned CNTs. ${ }^{34}$

Previous reports show that high temperature conventional bulk treatments performed in $\mathrm{Ar}$ and $\mathrm{N}_{2}$ atmospheres lead to graphitization of CNTs, ${ }^{4,35,36}$ though above a temperature threshold the creation of structural defects is also observed. ${ }^{37}$ As a consequence, the disorder-induced Raman band's intensity decreases leading to a reduction of $D / G$ and $\mathrm{D}^{\prime} / \mathrm{G}$ ratios. Figure 2 shows that in the case of laser irradiation of MWCNTs, beyond a laser fluence threshold value the $\mathrm{D} / \mathrm{G}$ and $\mathrm{D}^{\prime} / \mathrm{G}$ ratios clearly decrease with the laser fluence and the number of accumulated pulses. Nevertheless, the mechanisms responsible of this behaviour are radically different to those of conventional annealing methods since electron microscopy characterizations undoubtedly show the formation of a higher number of defects and even the melting of the CNTs (Figures 1, 4-6). It should be noticed that visible Raman spectroscopy is much more sensitive to $\mathrm{sp}^{2}$ than $\mathrm{sp}^{3}$ sites since visible photons (as used in our analyses) preferentially excite $\pi$ states. Thus, the obtained Raman spectra would mainly depend on the ordering of $\mathrm{sp}^{2}$ sites. On the other hand, the observed $\mathrm{G}$ mode, characteristic of graphite, involves the in-plane bond-stretching motion of $\mathrm{sp}^{2}$ carbon atoms. Thus, all $\mathrm{sp}^{2}$ sites contribute to this mode and not only those of sixfold rings. ${ }^{38}$ Furthermore, D peak is related to A1g symmetry breathing mode and arises from the presence of defects in aromatic rings. Therefore, the observed 
reduction of $\mathrm{D} / \mathrm{G}$ and $\mathrm{D}^{\prime} / \mathrm{G}$ ratios with the laser fluence and the number of accumulated pulses could be ascribed to a higher yield of decomposition of carbon aromatic rings, as compared with $\mathrm{sp}^{2}$ atoms which could be present in form of chains or disordered clusters. The observation of considerable melting of MWCNTs in the experimental conditions in which $D / G$ and $D^{\prime} / G$ ratios significantly decrease also supports this explanation.

Electron microscopy investigations clearly show that laser irradiation with low laser fluences provokes the formation of protuberances and quasi-spherical droplets at different separated locations of the CNTs (Figures 1(c) and 1(d); 4(b)). Many of the bulges observed by HRTEM are composed of amorphous material, opposite to the typical clusters observed after IR continuous wave laser irradiation of MWCNTs which show crystalline structure. ${ }^{39}$ Previous works showed that crystalline defects can produce a localized loss of atomic order in the material surrounding them at temperatures below the bulk transition, ${ }^{40}$ leading to the so called premelting process. In our case, the crystalline defects contained in the pristine MWCNTs would probably act as nucleation sites for the melting process at temperatures well below the one for "bulk" CNTs. ${ }^{27}$ Moreover, simple C-C bonds probably included in crystalline defects, with dissociation energy lower than the used photon energy, would promote local melting processes via photothermal and photochemical mechanisms. For these reasons, the protuberances mainly appear in CNTs irradiated with laser fluences below $80 \mathrm{~mJ} \mathrm{~cm} \mathrm{~cm}^{-2}$ where an extensive melting process of the "bulk" CNTs start to be detected even with low number of accumulated pulses. The accumulation of laser pulses would lead to the propagation of crystalline defects and the concomitant melting and amorphization of larger extension of the CNTs. Interestingly, the phase transition from liquid carbon to tetrahedral amorphous carbon in high pressure and supercooling conditions has been recently reported. ${ }^{41}$ As observed in the thermal simulations, laser irradiations would be able to provoke extremely high temperatures and cooling rates (Figure 7(b)) compatible to supercooling processes for the formation of amorphous carbon phase. Furthermore, taking into account the observed formation of nanodiamonds (Figure 5) along with the absence of vaporization processes in the molten regions, the development of not only high temperatures but also very high pressures inside the CNTs is expected during the heating process. ${ }^{42}$ Previous investigations revealed that carbon nanotubes can transform to diamond under different processing conditions, such as chemical vapour deposition (by nanotube coating), ${ }^{43}$ shock wave, ${ }^{44}$ direct transformation under high pressures and high temperatures, ${ }^{45}$ electron beam irradiation, ${ }^{46}$ and laser processing by means of continuous wave IR radiation. ${ }^{47}$ In the last two methods, the mechanism of transformation from carbon nanotubes to diamond was proposed to go through the formation of an intermediate form of carbon onions. The nucleation and growth of diamond crystals in the centre of the spherical carbon onions was identified to be critical. However, in our work performed with fast pulsed UV laser radiation, no carbon onions have been identified and the diamond nanocrystals, frequently immersed in amorphous matrix, seem to nucleate from molten carbon. It is also important to mention that high-pressure metastable phases, such as diamond, can be obtained at lower pressure when the material size reduces to the nanometer scale. ${ }^{48}$ Essentially, phase-transition mechanisms of nanocarbon strongly depend on carbon size, and diamond nanoclusters below a critical dimension can reveal higher stability than nanographite at ambient conditions. ${ }^{49,50}$

On the other hand, extensive melting of MWCNTs produces the sintering between them. As a result, the accumulation of a high enough number of laser pulses leads to the formation of a continuous solid film by means of meltingresolidification cycles (Figure 1(f)). The certainly low intensity of the Raman bands recorded in the continuous film accounts for its highly amorphous nature. Moreover, the slightly higher D/G ratio as well as shift to higher wavenumbers of $\mathrm{G}$ mode observed when comparing the Raman spectra inside and outside a MWCNT island surrounded by the continuous film (Figure 3) could be attributed to the contribution of relatively large regions with graphite structure inside the CNTs and graphite-like nanoclusters immersed in the amorphous matrix in the compact film. ${ }^{38}$ Numerical calculations also suggest that continuous and compact films could experience less extreme thermal cycles (Figures 7(b) and 7(c)) than isolated CNT structures. Thus, lower temperature-pressure and supercooling conditions would be achieved leading to the nucleation of nanocrystalline graphite rather than diamond. ${ }^{49,50}$

MWCNTs have a negative coefficient of thermal expansion along their main axis ${ }^{51}$ and, therefore, tend to shrink along their principal axis when heated. In addition, the thermal conductivity of nanocarbon can highly vary depending on the allotrope form ${ }^{52}$ leading to the development of extremely high thermal gradients in reduced regions with different structure inside the CNTs. Moreover, thermal expansion mechanism can be significantly altered with the presence of intrinsic defect sites which would induce localized thermal mismatch. Therefore, the rapid thermal cycling provoked by pulsed laser irradiation below the melting threshold could produce the corrugation of pristine MWCNTs due to excessive accumulated stress confined in tens of $\mathrm{nm}$ regions (Figures 6(a) and 6(b)). Indeed, the high pressure values developed in the MWCNTs due to all these mechanisms could be the main responsible for the melting of the CNTs without extensive vaporization and the formation of highpressure phases, such as diamond. Previous works also point out to different structural defects formed by UV laser irradiation in vacuum, such as amorphization, of the outer MWCNT shells and the formation of nanometer sized fragments in the inner hollow cavity of CNTs, probably caused by the migration of carbon atoms inside the nanotubes. ${ }^{14,53}$ The UV laser irradiation of double-walled CNTs in Ar ambient led to the formation of multi-walled carbon nanofibers, hollow, and solid onion-like nanoparticles. ${ }^{11}$ Finally, reported UV laser irradiation of CNTs in air $^{12}$ reveals the oxidation of the nanotubes' structure which transforms to cauliflower-like form. These facts certainly account for the great importance of the environment during the irradiation process since it is a determinant factor for the chemical reaction pathway. 


\section{SUMMARY}

Laser irradiation of multi-walled carbon nanotubes network-films deposited on Si substrates has been performed using nanosecond laser pulses with $266 \mathrm{~nm}$ wavelength. The treatments are carried out in nitrogen gas environment in order to avoid oxidation reactions. Numerical simulations indicate that the laser radiation can provoke thermal cycles with durations in the range of tens of ns. Depending on the mesh configuration, the CNTs can reach up to thousands of Kelvins, even surpassing their melting point. The evolution of the MWCNT morphology, structure, and composition with the accumulation of laser pulses shows that UV laser irradiation produces the formation of different nanocarbon forms. At low laser fluences the structural change begins at defects sites, leading to their premelting and formation of amorphous and even nanodiamond forms after cooling. In particular, the formation of diamond nanocrystals without the creation of intermediate carbon onions points to the development of very high temperature and pressure inside nanosized regions of the CNTs. The increase in the laser fluence and accumulation of pulses leads to the total melting and amorphization of the MWCNTs. The structure variation observed in MWCNTs differs from that of conventional high temperature annealing treatments. Thus, this work demonstrates that UV laser irradiation is an interesting technique that allows a fast and versatile modification of MWCNT sheets with tailored properties.

\section{ACKNOWLEDGMENTS}

This work was supported by the Spanish National Research Council under the Contract Nos. 200960I015, 200860I211, and MAT2011-24757; the EU FP7-ITN RADDEL No. 290023; and the Executive Unit for Financing Higher Education, Research, Development, and Innovation of the Romanian Ministry of Education, Research, Youth, and Sports under the Contract No. PCE-2012-4-0292. The authors are grateful to Thomas Swan Co. Ltd. for supplying CNT samples. L.C. acknowledges a JAE-Predoc fellowship (CSIC). L.C. is involved in the UAB PhD program. Authors also want to thank Dr. Elena Bailo from WITec for carrying out measurements with confocal Raman microscopy.

${ }^{1}$ M. F. L. De Volder, S. H. Tawfick, R. H. Baughman, and A. J. Hart, Science 339, 535 (2013).

${ }^{2}$ A. Salehi-Khojin, F. Khalili-Araghi, M. A. Kuroda, K. Y. Lin, J. P. Leburton, and R. I. Masel, ACS Nano 5, 153 (2011).

${ }^{3}$ Y. Wang and J. T. W. Yeow, J. Sensors 2009, 493904.

${ }^{4}$ E. Castillejos, B. Bachiller-Baeza, M. Pérez-Cadenas, E. Gallegos-Suarez, I. Rodríguez-Ramos, A. Guerrero-Ruiz, K. Tamargo-Martinez, A. Martinez-Alonso, and J. M. D. Tascón, J. Alloys Compd. 536, S460 (2012).

${ }^{5}$ D. Bäuerle, Laser Processing and Chemistry (Springer Verlag, Berlin, 2000).

${ }^{6}$ F. C. Cheong, K. Y. Lim, C. H. Sow, J. Lin, and C. K. Ong, Nanotechnology 14, 433 (2003).

${ }^{7}$ S. K. Chang-Jian, J. R. Ho, and J. W. J. Cheng, Appl. Phys. A 105, 691 (2011).

${ }^{8}$ H. Huang, R. Maruyama, K. Noda, H. Kajiura, and K. Kadono, J. Phys. Chem. B 110, 7316 (2006).

${ }^{9}$ X. Bai, D. Li, D. Du, H. Zhang, L. Chen, and J. Liang, Carbon 42, 2125 (2004).

${ }^{10}$ M. Q. Tran, C. Tridech, A. Alfrey, A. Bismarck, and M. S. P. Shaffer, Carbon 45, 2341 (2007).
${ }^{11}$ Y. Zhang, T. Gong, J. Wei, W. Liu, K. Wang, and D. Wu, J. Phys. Chem. C 111, 2901 (2007).

${ }^{12}$ P. D. Kichambare, L. C. Chena, C. T. Wang, K. J. Ma, C. T. Wu, and K. H. Chen, Mater. Chem. Phys. 72, 218 (2001).

${ }^{13}$ A. Perez del Pino, E. György, L. Cabana, B. Ballesteros, and G. Tobias, Carbon 50, 4450 (2012).

${ }^{14}$ E. György, A. Pérez del Pino, J. Roqueta, B. Ballesteros, L. Cabana, and G. Tobias, J. Nanopart. Res. 15, 1852 (2013).

${ }^{15}$ G. Tobias, L. Shao, C. G. Salzmann, Y. Huh, and M. L. H. Green, J. Phys. Chem. B 110, 22318 (2006).

${ }^{16}$ B. Ballesteros, G. Tobias, L. Shao, E. Pellicer, J. Nogués, E. Mendoza, and M. L. H. Green, Small 4, 1501 (2008).

${ }^{17}$ J. L. Bahr, E. T. Mickelson, M. J. Bronikowski, R. E. Smalley, and J. M. Tour, Chem. Commun. 2, 193 (2001).

${ }^{18}$ H. Murphy, P. Papakonstantinou, and T. I. T. Okpalugo, J. Vac. Sci. Technol. B 24, 715 (2006).

${ }^{19}$ S. Maldonado, S. Morin, and K. J. Stevenson, Carbon 44, 1429 (2006).

${ }^{20}$ A. Cuesta, P. Dhamelincourt, J. Laurenys, A. Martinez-Alonso, and J. M. D. Tascón, Carbon 32, 1523 (1994).

${ }^{21}$ G. W. Yang, J. B. Wang, and Q. X. Liu, J. Phys.: Condens. Matter 10, 7923 (1998).

${ }^{22}$ A. Vogel and V. Venugopalan, Chem. Rev. 103, 577 (2003).

${ }^{23}$ X. Huang, J. Wang, G. Eres, and X. Wang, Carbon 49, 1680 (2011).

${ }^{24}$ D. M. Haaland, Carbon 14, 357 (1976).

${ }^{25}$ S. Berber, Y. K. Kwon, and D. Tománek, Phys. Rev. Lett. 84, 4613 (2000).

${ }^{26}$ Z. Márton, B. Hopp, Z. Kántor, G. Sáfrán, G. Radnóczi, O. Geszti, and P. Heszler, App. Surf. Sci. 168, 154 (2000).

${ }^{27}$ K. Zhang, G. M. Stocks, and J. Zhong, Nanotechnology 18, 285703 (2007).

${ }^{28}$ A. Chehrghani, M. J. Torkamany, M. J. Hamedi, and J. Sabbaghzadeh, Appl. Surf. Sci. 258, 2068 (2012).

${ }^{29}$ T. Nakamiya, T. Ueda, T. Ikegami, F. Mitsugi, K. Ebihara, Y. Sonoda, Y. Iwasaki, and R. Tsuda, Thin Solid Films 517, 3854 (2009).

${ }^{30}$ A. E. Aliev, C. Guthy, M. Zhang, S. Fang, A. A. Zakhidov, J. E. Fischer, and R. H. Baughman, Carbon 45, 2880 (2007).

${ }^{31}$ A. E. Aliev, M. H. Lima, E. M. Silverman, and R. H. Baughman, Nanotechnology 21, 035709 (2010).

${ }^{32}$ A. Pérez del Pino, E. György, I. C. Marcus, J. Roqueta, and M. I. Alonso, Nanotechnology 22, 295304 (2011).

${ }^{33}$ T. Pichler, M. Knupfer, M. S. Golden, A. Rinzler, R. E. Smalley, and J. Fink, Phys. Rev. Lett. 80, 4729 (1998).

${ }^{34}$ T. Nakamiya, F. Mitsugi, K. Semba, R. Kozai, T. Ikegami, Y. Iwasaki, Y. Sonoda, and R. Tsuda, Thin Solid Films 518, 6604 (2010).

${ }^{35}$ Y. A. Kim, T. Hayashi, K. Osawa, M. S. Dresselhaus, and M. Endo, Chem. Phys. Lett. 380, 319 (2003).

${ }^{36}$ R. Andrews, D. Jacques, D. Qian, and E. C. Dickey, Carbon 39, 1681 (2001).

${ }^{37}$ J. Zhao, Y. Zhang, Y. Su, X. Huang, L. Wei, E. S. W. Kong, and Y. Zhang, Diamond Relat. Mater. 25, 24 (2012).

${ }^{38}$ A. C. Ferrari and J. Robertson, Phys. Rev. B 61, 14095 (2000).

${ }^{39}$ R. Z. Ma, B. Q. Wei, C. L. Xu, J. Liang, and D. H. Wu, Carbon 38, 636 (2000).

${ }^{40}$ A. M. Alsayed, M. F. Islam, J. Zhang, P. J. Collings, and A. G. Yodh, Science 309, 1207 (2005).

${ }^{41}$ D. R. Robinson and M. Wilson, J. Phys.: Condens. Matter. 25, 155101 (2013).

${ }^{42}$ M. P. Grumbach and R. M. Martin, Phys. Rev. B 54, 15730 (1996).

${ }^{43}$ Y. Q. Hou, D. M. Zhuang, G. Zhang, M. S. Wu, and J. J. Liu, Appl. Surf. Sci. 185, 303 (2002).

${ }^{44}$ Y. Q. Zhu, T. Sekine, T. Kobayashi, T. Takazawa, M. Terrones, and H. Terrones, Chem. Phys. Lett. 287, 689 (1998).

${ }^{45}$ L. Cao, C. Gao, H. Sun, G. Zou, Z. Zhang, X. Zhang, M. He, M. Zhang, Y. Li, J. Zhang, D. Dai, L. Sun, and W. Wang, Carbon 39, 311 (2001).

${ }^{46}$ A. V. Krasheninnikov and F. Banhart, Nature Mater. 6, 723 (2007).

${ }^{47}$ B. Wei, J. Zhang, J. Liang, and D. Wu, Carbon 36, 997 (1998).

${ }^{48}$ C. X. Wang and G. W. Yang, Mater. Sci. Eng., R 49, 157 (2005).

${ }^{49}$ C. C. Yang and S. Li, J. Phys. Chem. C 112, 1423 (2008).

${ }^{50}$ O. A. Shenderova, V. V. Zhirnov, and D. W. Brenner, Crit. Rev. Solid State Mater Sci. 27, 227 (2002).

${ }^{51}$ Y. K. Kwon, S. Berber, and D. Tománek, Phys. Rev. Lett. 92, 015901 (2004).

${ }^{52}$ A. A. Balandin, Nature Mater. 10, 569 (2011).

${ }^{53}$ G. Singh, P. Rice, K. E. Hurst, J. H. Lehman, and R. L. Mahajan, Appl. Phys. Lett. 91, 033101 (2007). 